\title{
Deferoxamine: potential novel topical therapeutic for chronic wounds
}

DOI: $10.1111 /$ bjd. 14956

Although chronic wounds have multifactorial aetiologies, they share a common characteristic of compromise of the local vasculature leading to diminished oxygen tension. ${ }^{1}$ In pressure ulcers (PU), the obstruction of capillary blood flow and lymphatics from the local shear pressure results in ischaemia. The subsequent reperfusion causes hyperaemia and oedema, further reducing the regional oxygen level. ${ }^{1}$ Similarly, in venous leg ulcers (VLU), vascular permeability and increase in venous hypertension generate local oedema. In diabetic foot ulcers (DFU), hypoxia results from local pressure and high metabolic stress on the wound bed. During the initial phase of healing for all three wound types, the local and acute hypoxic climate results in the release of numerous growth factors by endothelial cells, fibroblasts and macrophages. ${ }^{1}$ One of those essential factors is the hypoxia inducible factor-1 (HIF-1). The regulation of HIF-1 activity resides in its oxygen-dependent degradation, by prolyl 4-hydroxylase (PHD) enzymes that require iron $\left(\mathrm{Fe}^{2+}\right)$ as a cofactor. Under hypoxic conditions, HIF- $1 \alpha$ is stabilized against PHD enzymatic degradation and moves to the nucleus, where it dimerizes with HIF-1 $\beta$ and binds to a hypoxia response element. For its transcriptional activity, HIF- $1 \alpha$ also needs to bind to the coactivators p300/cyclic adenosine monophosphate response element binding protein (CREB) binding protein (CBP). ${ }^{1}$ This results in the upregulation and the activation of more than 60 HIF-1 $\alpha$ target genes for tissue repair, cell growth and proliferation, and important angiogenic factors such as vascular endothelial growth factor (VEGF; Fig. 1). ${ }^{1}$ Although VEGF induces angiogenesis, studies have shown that prolonged hypoxia results in increased acidosis, decrease adenosine triphosphate production and inhibition of neovascularization that cannot be reversed by VEGF. ${ }^{1}$ This explains the need for a therapeutic agent that would maintain the multitarget activity of HIF-1 $\alpha$ within the chronic wound environment (Fig. 1).

Another characteristic of chronic wounds is the local deposition of free iron. In VLU, leakage of erythrocytes into the interstitium and their degradation by macrophages leads to the release of the iron bound to ferritin. In patients with diabetes, insulin resistance results in the increase in ferritin synthesis and iron stores, which concurrently reduce the hepatic extraction and metabolism of insulin, leading to hyperinsulinaemia. ${ }^{2}$ We speculate that the local tissue ischaemia with vessel occlusion encountered in PU also leads to increased iron deposition (Fig. 1).

Iron is a transition metal that has the ability to donate or accept single electrons. This allows iron to neutralize free radicals and thus to serve as a critical antioxidant defence. ${ }^{2}$ In order to maintain the physiological redox status of the cells, iron is sequestered bound with either proteins or cofactors. ${ }^{2}$ If released from its bound form, free iron serves as a chemoattractant for neutrophils and macrophages. The combination of iron and hydrogen peroxide (from activated neutrophils) generates ferric ion $\left(\mathrm{Fe}^{3+}\right)$ and reactive oxygen species (ROS), the prolonged production of which results in a chronically inflamed environment. ${ }^{1}$ A vicious cycle is created by the reduction of ferric to ferrous ion $\left(\mathrm{Fe}^{2+}\right)$, generating more ROS, and the subsequent ROS enhancement of release of iron from carriers (Fig. 1). ${ }^{1}$ Iron also induces an unrestrained proinflammatory M1 macrophage phenotype, which contributes to perpetual tissue damage and compromised tissue regeneration as the cells fail to switch to the proreparative M2 phenotype. ${ }^{3}$ All of the above sustain the chronic inflammatory state encountered in chronic wounds (Fig. 1).

We postulate that the topical application of deferoxamine (DFO), which simultaneously acts as an iron chelator and effective HIF- $1 \alpha$ inducer and stabilizer, is a potential therapeutic approach to improve healing of chronic wounds. DFO could be topically administered to the impaired wound bed at concentrations 100-fold lower than the therapeutic parenteral infusion warranted in the treatment of thalassaemia or iron poisoning, and with a better safety profile.

Preclinical studies have demonstrated that DFO topically applied or injected improved wound healing in $\mathrm{db} / \mathrm{db}$ mice, with increased granulation tissue and neovascularization. ${ }^{1,4}$ DFO modulated the expression of several cytokines and growth factors (including HIF-1 $\alpha$ and VEGF). ${ }^{4}$ As a result, $\mathrm{DFO}$ led to decreased inflammation, enhanced angiogenesis and wound maturation. The authors proposed that DFO ointment could potentially heal cutaneous wounds in patients with diabetes. ${ }^{4}$ The addition of DFO to fibroblasts derived from diabetic $\mathrm{db} / \mathrm{db}$ mice cultured in hyperglycaemic and hypoxic conditions resulted in increased expression of HIF-1 $\alpha$ target genes responsible for motility and angiogenesis. ${ }^{1}$ DFO enhanced wound healing rates in the $\mathrm{db} / \mathrm{db}$ mouse, even in the presence of continuous hyperglycaemia. ${ }^{1}$

Human aortic endothelial cells cultured in high glucose and hypoxia with DFO, resulted in a twofold elevation in HIF- $1 \alpha$ binding to p300 coactivator, approaching levels found in cells cultivated in low glucose. ${ }^{1}$ DFO prevented the methylglyoxal modification of p300, which has been shown to impact negatively on the heterodimerization of the two HIF-1 subunits. $^{1}$ Additionally, local injection of DFO into ischaemic flaps of diabetic mice resulted in wound healing enhancement 


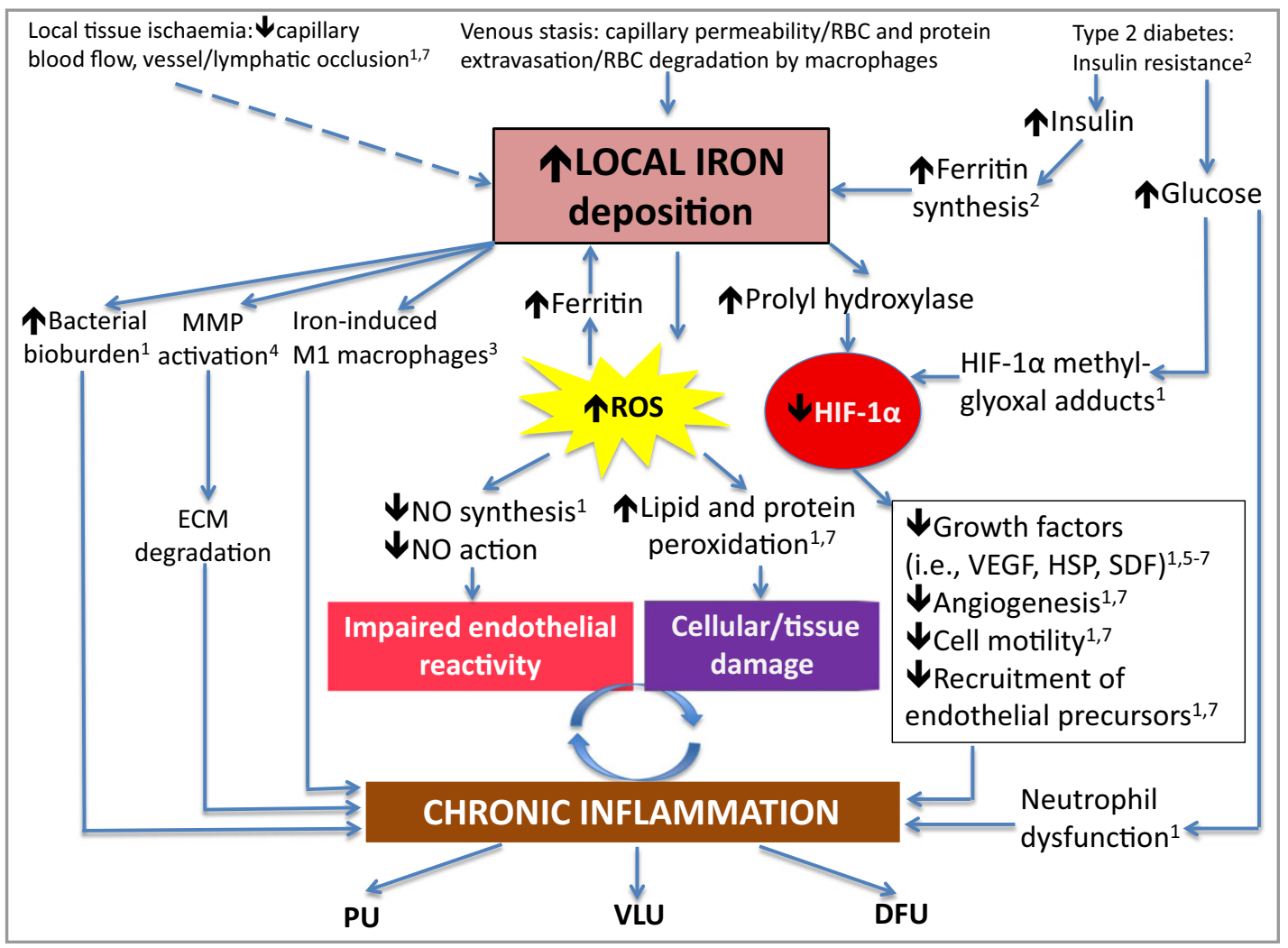

Fig 1. Schematic role of iron in the pathogenesis of chronic wound formation. RBC, red blood cells; MMP, matrix metalloprotease; ROS, reactive oxygen species; HIF-1 $\alpha$, hypoxia-inducible factor-1; ECM, extracellular matrix; NO, nitric oxide; VEGF, vascular endothelial growth factor; HSP, heat shock protein; SDF, stromal-derived factor; PU, pressure ulcer; VLU, venous leg ulcer; DFU, diabetic foot ulcer.

and prevention of flap necrosis by increasing HIF-1 $\alpha$ and VEGF expression, endothelial cell recruitment and neovascularization. ${ }^{5}$

A very different approach by Duscher et al. ${ }^{6}$ demonstrated transdermal drug delivery of DFO in reverse micelles that penetrate the intact stratum corneum. This transdermal delivery system improved PU healing and prevented ulcer formation in diabetic mice. The authors suggested that this DFO delivery system could prevent diabetic ulcers and improve wound healing in humans. ${ }^{6}$

Our (C.N.T.-F. and S.R.B.) translational experiments in radiation-induced delayed bone healing have led us and others to investigate $\mathrm{DFO}$ as a therapeutic option to optimize tissue repair and regeneration. ${ }^{7}$ We used a murine mandibular model of distraction osteogenesis (DO) and pathological fracture in the setting of radiation. DO is a reconstructive technique that leads to endogenous bone regeneration after the separation of two osteogenic fronts. In these models DFO increased the vascular metrics compared with radiated untreated controls. ${ }^{7}$ All of these aforementioned studies demonstrate that DFO improves bony tissue repair, even in the absence of hyperglycaemia-induced deficits. Although both skin and bone are dynamic organs with different repair mechanisms, our findings corroborate those of others in the context of delayed wound healing.
Clinical studies have correlated the severity of VLU with the abundance of iron in the wound dermis. ${ }^{3,8}$ They also revealed sizable amounts of iron in chronic wound exudates of VLU compared with acute wound exudates. ${ }^{3,8}$

In vivo studies of patients with diabetes have shown that hyperglycaemia impairs transferrin binding ability and augments ferritin synthesis, ${ }^{2}$ thus increasing the availability of free iron and promoting ROS formation. In addition, hyperglycaemia interferes in a dose-dependent manner with HIF-1 $\alpha$ protein stabilization, ${ }^{1}$ resulting in low levels found in diabetic wounds, with a concomitant decrease in angiogenesis contributing to wound chronicity.

One of the earliest human studies linking DFO and glycaemic control in patients with diabetes was done in 1989 . $^{2}$ Following intravenous DFO administration, patients with diabetes with high serum ferritin no longer required hypoglycaemic therapy. ${ }^{2}$ Thus, studying DFO therapy in clinical trials may decrease diabetic complications, such as DFU. ${ }^{6}$ Most other human clinical studies have focused on DFO's protective effect on the myocardium against reperfusion-induced free radical formation in cardiac patients, or in the prevention of iron-induced heart failure and arrhythmia in thalassaemia., 2,6 In addition, DFO has been examined as a preventative measure for intracerebral haemorrhage and therefore ischaemic stroke as it counteracts iron-mediated neurotoxicity by decreasing 
cerebral oedema and neuronal death. ${ }^{6}$ However, to date there are no human studies that have investigated the clinical application of DFO for the treatment of any type of chronic wound.

Given the hypoxia-driven environment of chronic wounds and the toxic effect of local iron deposition, it is fair to postulate that a topical delivery of DFO could reverse those deleterious effects and make a significant contribution to wound healing. Pharmacokinetic studies have shown that DFO is highly hydrophilic and rapidly metabolized by plasma enzymes, ${ }^{6,9}$ which leads to a short half-life of 12 min. ${ }^{6}$ Therefore, there is decreased absorption by the gastrointestinal tract and limited efficacy. Consequently, DFO has preferentially been administered subcutaneously for the treatment of iron overload.

DFO is usually well tolerated, and adverse reactions to DFO have been insufficiently reported to quantify their frequency. At high doses (exceeding $40 \mathrm{mg} \mathrm{kg}^{-1}$ daily) subcutaneous administration of DFO may produce pain and swelling at the injection site, ocular, auditory, neurological disturbances and renal insufficiency, with the latter shown to reverse upon discontinuation of DFO therapy. ${ }^{9}$ Hypotension, growth retardation in children and opportunistic infections, (in particular, mucormycosis in patients on dialysis) have also been reported. ${ }^{9}$ Severe pulmonary effects have been observed at intravenous infusion doses of $10-20 \mathrm{mg} \mathrm{kg}^{-1}$ hourly. ${ }^{9}$ As far as the skin is concerned, a very rare generalized rash has been described. ${ }^{6,9}$ Several randomized clinical trials on the use of iron chelators for the treatment of thalassaemia have shown that DFO has a good safety profile compared with other chelators. There have not been any reactions reported from the topical use of DFO.

Here we propose that DFO is administered topically at concentrations 100-fold lower than the therapeutic dosage used parenterally in thalassaemia or iron poisoning, thus with a better safety profile. A major potential barrier to penetration of topically administered DFO is the stratum corneum, the outermost layer of the skin. However, chronic wounds lack the intact stratum corneum. In addition, numerous studies have now documented that DFO uptake does not occur via permeation across cellular membrane but rather via fluidphase endocytosis, leading to an exclusive intracellular location within the endosome-lysosome complex. ${ }^{10}$ As the stratum corneum is absent in chronic wounds and DFO absorption occurs through an endosomal pathway, we anticipate easy delivery of $\mathrm{DFO}$ and a direct absorption at the local wound site, including weight-bearing areas such as plantar DFU.

We envision that DFO can be administered topically as a solution, cream or medication delivery patch directly on the wound bed. DFO would be readily available at the local wound site as it would avoid the first pass by the liver.

The topical use of DFO as an iron chelator seems to have multiple potential advantages. DFO can prevent iron release in the tissue of patients with diabetes, or venous or PUs and therefore indirectly inhibit the deleterious cascade of ironinduced oxygen free radicals that overload the wound's antioxidant capacities and perpetuate the inflammatory damage cycle. Furthermore, DFO-induced HIF- $1 \alpha$ stabilization would circumvent the chronic hypoxic milieu of PU, VLU and DFU, and stimulate new vessels, potentiate cell motility and recruitment of endothelial precursors, all of which are essential to the healing phase of cutaneous wounds. Altogether, DFO has the potential to provide a novel therapeutic tool to improve healing in chronic wounds.

\section{Acknowledgments}

We wish to acknowledge the work of Thi (Tina) Dinh La, who helped us with the illustration of our hypothesis.

\section{Conflicts of interest}

None declared.

\author{
${ }^{1}$ Department of Dermatology, University C.N. TCHANQUe-Fossuo ${ }^{1,2}$ \\ of California Davis, 3301 C Street, \\ S.E. Dahle ${ }^{1,3}$ \\ Sacramento, CA, U.S.A. \\ ${ }^{2}$ Veterans Administration, Northern \\ S.R. BuCHMAN ${ }^{4}$ \\ California Health Care System, Dermatology \\ Service, 10535 Hospital Way, Building \\ 801, Mather, CA 95655, U.S.A. \\ ${ }^{3}$ Department of Surgery, Podiatry Section, \\ 10535 Hospital Way, Building 801, \\ Mather, CA 95655, U.S.A. \\ ${ }^{4}$ Plastic Surgery Section, University of \\ Michigan, Ann Arbor, MI, U.S.A. \\ E-mail: tchanquec@gmail.com
}

\section{References}

1 Glotzbach JP, Wong VW, Gurtner GC. Neovascularization in diabetes. Expert Rev Endocrinol Metab 2010; 5:99-111.

2 Fernandez-Real JM, Lopez-Bermejo A, Ricart W. Cross-talk between iron metabolism and diabetes. Diabetes 2002; 51:2348-54.

3 Sindrilaru A, Peters T, Wieschalka S et al. An unrestrained proinflammatory M1 macrophage population induced by iron impairs wound healing in humans and mice. J Clin Invest 2011; 121:98597.

4 Ram M, Singh V, Kumawat S et al. Deferoxamine modulates cytokines and growth factors to accelerate cutaneous wound healing in diabetic rats. Eur J Pharmacol 2015; 764:9-21.

5 Wang C, Cai Y, Zhang Y et al. Local injection of deferoxamine improves neovascularization in ischemic diabetic random flap by increasing HIF-1 $\alpha$ and VEGF expression. PLOS ONE 2014; 9: e100818.

6 Duscher D, Neofytou E, Wong VW et al. Transdermal deferoxamine prevents pressure-induced diabetic ulcers. Proc Natl Acad Sci USA 2015; 112:94-9.

7 Donneys A, Weiss DM, Deshpande SS et al. Localized deferoxamine injection augments vascularity and improves bony union in pathologic fracture healing after radiotherapy. Bone 2013; 52:318-25.

8 Wenk J, Foitzik A, Achterberg $\mathrm{V}$ et al. Selective pick-up of increased iron by deferoxamine-coupled cellulose abrogates the iron-driven induction of matrix-degrading metalloproteinase 1 
and lipid peroxidation in human dermal fibroblasts in vitro: a new dressing concept. J Invest Dermatol 2001; 116:833-9.

9 Novartis. Desferal R/Deferoxamine Mesylate for Injection. East Hanover, NJ: Novartis, 2011.
10 Doulias PT, Christoforidis S, Brunk UT, Galaris D. Endosomal and lysosomal effects of desferrioxamine: protection of HeLa cells from hydrogen peroxide-induced DNA damage and induction of cellcycle arrest. Free Radic Biol Med 2003; 35:719-28. 